\title{
ON KAN-INJECTIVITY OF LOCALES AND SPACES
}

\author{
MARGARIDA CARVALHO AND LURDES SOUSA
}

\begin{abstract}
Aвstract. In the category $\mathrm{Top}_{0}$ of $T_{0}$-spaces and continuous maps, embeddings are just those morphisms with respect to which the Sierpiński space is Kan-injective, and the Kaninjective hull of the Sierpiński space is the category of continuous lattices and maps preserving directed suprema and arbitrary infima. In the category Loc of locales and localic maps, we give an analogous characterization of flat embeddings; more generally, we characterize $n$-flat embeddings, for each cardinal $n$, as those morphisms with respect to which a certain finite subcategory is Kan-injective. As a consequence, we obtain similar characterizations of the $n$-flat embeddings in the category $\mathrm{Top}_{0}$, and we show that several well-known subcategories of Loc and $\mathrm{Top}_{0}$ are Kan-injective hulls of finite subcategories. Moreover, we show that there is a subcategory of spatial locales whose Kan-injective hull is the entire category Loc.
\end{abstract}

\section{INTRODUCTION}

Two well-known important facts on injectivity are the characterization of the continuous lattices as the spaces injective with respect to the embeddings in the category Top of $T_{0}$-spaces ([27]), and the characterization of the stably locally compact locales as the locales injective with respect to the flat embeddings in the category Loc of locales $([16,18])$. In $[7,8]$ Escardó pointed out that in many examples, including the above two, more than injectivity, we have Kan-injectivity (see also $[10,9]$ ). In an order-enriched category, we say that an object $A$ is left Kan-injective with respect to a morphism $h: X \rightarrow Y$ if every morphism $f: X \rightarrow A$ has a left Kan extension along $h$, denoted by $f / h$, and moreover, $(f / h) h=f$. In [5] we extended the concept of Kan-injectivity to morphisms: a morphism is left Kan-injective with respect to $h$ if it preserves left Kan extensions along $h$. This way (left) Kan-injectivity plays for KZ-monads (i.e., Kock-Zöberlein monads [21, 34], also called lax idempotent monads) the same role as orthogonality plays for idempotent monads, that is, Kan-injectivity may be regarded as a "lax" generalization of orthogonality. Indeed, in [5] and [3] several well-known results on orthogonality were recaptured in this more general environment in the scope of order-enriched categories. In particular,

Date: 16 April, 2015 (submitted); 28 September, 2015 (revised).

1991 Mathematics Subject Classification. 06D22, 18D20, 54B30.

Key words and phrases. Locales, topological spaces, injectivity, Kan extensions, KZ-monads.

The first author acknowledges partial support by FCT - Fundação para a Ciência e a Tecnologia under the project SFRH/PROTEC/67529/2010 and by ISCAC - IPC.

The second author acknowledges the support of the Centre for Mathematics of the University of Coimbra (funded by the program COMPETE and by the Fundação para a Ciência e a Tecnologia, under the project PEst-C/MAT/UI0324/2013). 
in [3] we gave an answer to the Kan-injective Subcategory Problem resembling the one of Freyd and Kelly [11] to the Orthogonal Subcategory Problem.

In this paper we concentrate on Kan-injectivity in the category Loc of locales and localic maps, and in the category $\mathrm{Top}_{0}$ of $T_{0}$ topological spaces and continuous maps. We show that embeddings, dense embeddings and flat embeddings may be characterized by means of a finite category via Kan-injectivity, both in Loc and in Top 0 . More generally, this is shown for $n$-flat embeddings, for every cardinal $n$ (with " 2 -flat" meaning just "flat"). That characterization of $n$-flat embeddings allows us to prove that several interesting subcategories of $\mathrm{Loc}$ and $\mathrm{Top}_{0}$ are Kan-injective hulls of finite subcategories. In particular, this applies to the subcategory of stably locally compact locales (with suitable morphisms) in Loc, and to the subcategory of continuous lattices (with suitable mor-

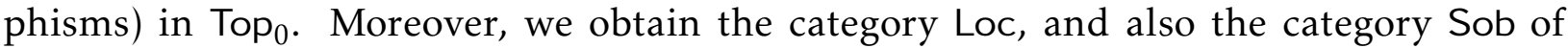
sober spaces, expressed as the union of an increasing chain of Kan-injective hulls of finite subcategories, which moreover, are KZ-monadic subcategories. In Loc, the objects of all these finite subcategories are spatial, and, as a consequence, Loc is the Kan-injective hull of a subcategory of spatial locales.

In [4], Bernard Banaschewski studied a general form of projectivity in the category of frames called $\mathcal{K}$-flat projectivity. Kan-injectivity of locales with respect to $n$-flat embeddings is a particular case of $\mathcal{K}$-flat projectivity of frames (see Remark 4.4.1).

\section{Preliminaries on Kan-Injectivity}

In this section we recall the general notions and results on Kan-injectivity in orderenriched categories, needed in the subsequent study on locales and spaces (cf. $[5,3]$ ).

Let Pos be the category of partially ordered sets and monotone maps. A category $X$ is said to be enriched in Pos, or just order-enriched, if every hom-set $X(X, Y)$ of all morphisms from $X$ to $Y$ is endowed with a partial order such that, for every pair of morphisms $f, g: X \rightarrow Y$, and morphisms $h: Z \rightarrow X$ and $k: Y \rightarrow W$, the inequality $f \leq g$ implies that $f h \leq g h$ and $k f \leq k g$. A functor $F: X \rightarrow y$ between order-enriched categories is said to be locally monotone, or order-enriched, if the inequality $f \leq g$ implies that $F f \leq F g$ for all morphisms $f$ and $g$ with common domain and codomain.

Definition 2.1. 1. Given an object $A$ and a morphism $h: X \rightarrow Y$ in an order-enriched category we say that $A$ is left Kan-injective with respect to $h$ if, for every morphism $f: X \rightarrow$ $A$,

(i) $f$ admits a left Kan-extension along $h$, that is, the set of all morphisms $s: Y \rightarrow A$ such that $f \leq s h$ has an infimum, which we denote by $f / h$; and 
(ii) $(f / h) h=f$.

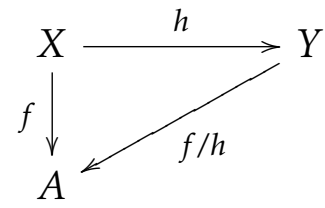

A morphism $g: A \rightarrow B$ is said to be left Kan-injective with respect to $h$ if the objects $A$ and $B$ are so, and $g$ satisfies the equality $g(f / h)=(g f) / h$ for every $f: X \rightarrow A$.

2. If in (i) we replace " $f \leq s h$ " with " $f \geq s h$ " and "infimum" with "supremum" we obtain the right Kan-injectivity concept.

Of course, if we reverse the order of the hom-sets of an order-enriched category, we get a new order-enriched category, and left Kan-injectivity in the former category becomes right Kan-injectivity in the latter, and vice-versa.

Along the paper we are going to work with left Kan-injectivity and we often omit the word "left".

In an order-enriched category a pair of morphisms $X \underset{l}{\stackrel{r}{\longleftarrow}} Y$ is said to be an adjoint pair, written $l \dashv r: X \rightarrow Y$, if $\mathrm{id}_{Y} \leq r l$ and $l r \leq \mathrm{id}_{X}$, being $r$ the right adjoint and $l$ the left adjoint. (We recall that $r$ and $l$ are mutually uniquely determined.) If, moreover, $l$ is a retraction, then it satisfies the equality $l r=\mathrm{id}_{X}$. Indeed, let $s: X \rightarrow Y$ be a right inverse of $l$; then $l r=l r l s \geq l s=\mathrm{id}_{X}$, and this, combined with $l r \leq \mathrm{id}_{X}$, gives $l r=\mathrm{id}_{X}$. When this is the case, we shall call $l$ a left adjoint retraction. Analogously, we shall use the expressions right adjoint retraction and left (or right) adjoint section with the obvious meaning.

Remark 2.2. We recall from Kock [21] and Escardó [7, 8] that a KZ-monad over an orderenriched category $X$ is a monad whose functor $F: X \rightarrow X$ is locally monotone and whose unit $\eta$ fulfils the condition

$$
F \eta_{X} \leq \eta_{F X} \text {, for all } X \in X
$$

Moreover, the structure map of each Eilenberg-Moore algebra of a KZ-monad is adjoint to the unit. More precisely, for $X \in X$ and $\alpha: F X \rightarrow X$, the pair $(X, \alpha)$ is an algebra if and only if we have an adjunction $\alpha \dashv \eta_{X}$ with $\alpha \eta_{X}=\mathrm{id}_{X}$, that is, $\eta_{X}$ is a right adjoint section; further, if it is the case, $\alpha=\mathrm{id}_{X} / \eta_{X}$.

Consequently, every object $X$ of $X$ is the structure of at most one Eilenberg-Moore algebra of the monad. Thus, categories of algebras of these monads can be seen, up to isomorphism of categories, as subcategories of $X$. These subcategories are said to be $K Z$ monadic ([3]).

In an order-enriched category $X$, given a subcategory $\mathcal{A}$, we write

$$
\mathcal{A}^{\mathrm{KInj}}
$$


to denote the class of all morphisms of $X$ with respect to which all objects and all morphisms of $\mathcal{A}$ are left Kan-injective. And, given a class $\mathcal{H}$ of morphisms of $\mathcal{X}$, taking all objects and morphisms which are left Kan-injective with respect to $\mathcal{H}$ we obtain a subcategory, designated by

$$
\operatorname{KInj}(\mathcal{H})
$$

and said to be a Kan-injective subcategory.

This way, (left) Kan-injectivity gives rise to a Galois connection between subcategories and classes of morphisms of $\mathcal{X}$. Thus, given a subcategory $\mathcal{A}$ of $\mathcal{X}$, the subcategory $\operatorname{KInj}\left(\mathcal{A}^{\mathrm{KInj}}\right)$ is the smallest Kan-injective subcategory of $\mathcal{X}$ containing $\mathcal{A}$. It will be called the Kan-injective hull of $\mathcal{A}$ and denoted by

$$
\mathbf{K}(\mathcal{A}) .
$$

The next theorem and proposition characterize KZ-monadic subcategories $\mathcal{A}$ and the corresponding class $\mathcal{A}^{\mathrm{KInj}}$. First we need the following definitions:

Definitions 2.3. 1. A subcategory $\mathcal{A}$ of an order-enriched category $\mathcal{X}$ is said to be closed under left adjoint retractions if, for every commutative diagram in $X$ of the form

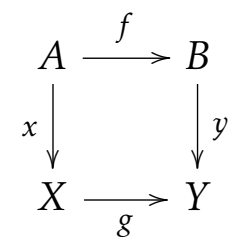

with $x$ and $y$ left adjoint retractions (as defined before Remark 2.2), if $f \in \mathcal{A}$ then also $g \in \mathcal{A}$.

2. Given a locally monotone functor $F: X \rightarrow y$, we call a morphism $f: X \rightarrow Y$ an $F$ embedding if $F f$ is a left adjoint section in $y$, i.e., if there is a morphism $(F f)_{*}: F Y \rightarrow F X$ with $(F f)_{*} F f=\operatorname{id}_{F X}$ and $F f(F f)_{*} \leq \operatorname{id}_{F Y}$.

Theorem 2.4. ([5]) Let $\mathcal{A}$ be a subcategory of the order-enriched category $X$. Then the following statements are equivalent:

(a) $\mathcal{A}$ is a KZ-monadic subcategory of $X$.

(b) $\mathcal{A}$ is a reflective subcategory of $\mathcal{X}$ closed under left adjoint retractions, whose reflector functor $F$ and unit $\eta$ satisfy the conditions:

(i) $\mathcal{A}=\operatorname{KInj}\left(\left\{\eta_{X} \mid X \in X\right\}\right)$, and

(ii) for every morphism $f: F X \rightarrow A$ in $\mathcal{A},\left(f \eta_{X}\right) / \eta_{X}=f$.

Remark 2.5. The condition (ii) above may be equivalently replaced with the following one: for every pair of morphisms $f, g: F X \rightarrow A$ with $f$ in $\mathcal{A}, f \eta_{X} \leq g \eta_{X}$ implies $f \leq g$.

Remark 2.6. In particular, Theorem 2.4 shows that every KZ-monadic subcategory is a Kan-injective subcategory, that is, of the form $\operatorname{KInj}(\mathcal{H})$, for some class $\mathcal{H}$ of morphisms. In [3] the authors present conditions on the base category under which the converse is 
true for all sets $\mathcal{H}$. The particular case of ordinary categories, enriched with the order equality, is then the solution for the Orthogonal Subcategory Problem given in $[11,19]$ (see also [1]).

Proposition 2.7. ([5]) Let $\mathcal{A}$ be a KZ-monadic subcategory of $X$ with $F: X \rightarrow \mathcal{A}$ and $I: \mathcal{A} \rightarrow X$ the reflector and inclusion functors, respectively. Then,

$$
\mathcal{A}^{\text {KInj }}=\{f \in X \mid f \text { is an F-embedding }\}=\{f \in X \mid f \text { is an IF-embedding }\},
$$

and $\mathcal{A}=\mathbf{K}(\mathcal{A})$.

We prove here another property of Kan-injectivity, which will be useful in the last section.

Proposition 2.8. Let $F \dashv G: \mathcal{A} \rightarrow X$ be an adjunction of locally monotone functors between order-enriched categories. Then, given a morphism h of $X$, an object $A$ (respectively, a morphism $f$ ) of $\mathcal{A}$ is Kan-injective with respect to $F h$ in $\mathcal{A}$ if and only if $G A$ (respectively, $G f$ ) is Kaninjective with respect to $h$ in $X$.

Remark. Before proving the proposition, we point out that, given the adjunction $F \dashv G$ : $\mathcal{A} \rightarrow X$ between order-enriched categories, the local monotonicity of the functors $G$ and $F$ is equivalent to the following condition for all pairs of morphisms $f, g: F X \rightarrow A$, where $\eta$ is the unit:

$$
G f \cdot \eta_{X} \leq G g \cdot \eta_{X} \Leftrightarrow f \leq g .
$$

Indeed, if $G$ and $F$ are locally monotone, one of the implications of (2) is obvious; and the less obvious one follows easily, since, given $f, g: F X \rightarrow A$ with $G f \cdot \eta_{X} \leq G g \cdot \eta_{X}$, then, using the counit $\varepsilon$ of the adjunction, we have $\varepsilon_{A} \cdot F G f \cdot F \eta_{X} \leq \varepsilon_{A} \cdot F G g \cdot F \eta_{X}$, thus $f \cdot \varepsilon_{F X} \cdot F \eta_{X} \leq g \cdot \varepsilon_{F X} \cdot F \eta_{X}$, that is, $f \leq g$. Conversely, assuming that condition (2) is satisfied, given $f, g: X \rightarrow Y$ in $X$ with $f \leq g$, we have $\eta_{Y} f \leq \eta_{Y} g$, that is, GFf $\cdot \eta_{X} \leq G F g \cdot \eta_{X}$, thus Ff $\leq F g$. Moreover, given $f, g: A \rightarrow B$ in $\mathcal{A}$ with $f \leq g$, then $f \varepsilon_{A} \leq g \varepsilon_{A}$, and, using condition (2), $G f \cdot G \varepsilon_{A} \cdot \eta_{G A} \leq G g \cdot G \varepsilon_{A} \cdot \eta_{G A}$, that is, $G f \leq G g$.

Proof. Let $G A$ be Kan-injective with respect to $h: X \rightarrow Y$. In order to prove that $A$ is Kan-injective with respect to $F h$, consider a morphism $u: F X \rightarrow A$. By hypothesis, there exists $\left(G u \cdot \eta_{X}\right) / h$. Let $\bar{u}$ be the unique morphism with $G \bar{u} \cdot \eta_{Y}=\left(G u \cdot \eta_{X}\right) / h$. We show that $u / F h=\bar{u}$.

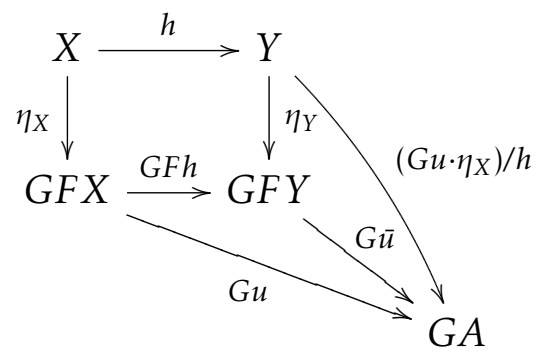


The equality $\bar{u} F h=u$ follows from the fact that we have $G(\bar{u} \cdot F h) \cdot \eta_{X}=G \bar{u} \cdot \eta_{Y} \cdot h=$ $\left(\left(G u \cdot \eta_{X}\right) / h\right) \cdot h=G u \cdot \eta_{X}$. Let now $s: F Y \rightarrow A$ be a morphism satisfying the inequality $u \leq s F h$. Then, by (2), Gu $\cdot \eta_{X} \leq G s \cdot G F h \cdot \eta_{X}=G s \cdot \eta_{Y} \cdot h$, and thus $\left(G u \cdot \eta_{X}\right) / h \leq G s \cdot \eta_{Y}$, that is, $G \bar{u} \cdot \eta_{Y} \leq G s \cdot \eta_{Y}$; using property (2) again, $\bar{u} \leq s$. Now it is easy to show the property also for morphisms: for $f: A \rightarrow B$ with $G f$ Kan-injective with respect to $h$, we will have $\left.G(f \bar{u}) \eta_{Y}=G f \cdot G \bar{u} \cdot \eta_{Y}=G f \cdot\left(\left(G u \cdot \eta_{X}\right) / h\right)=\left(G(f u) \cdot \eta_{X}\right) / h\right)=G \overline{f u} \cdot \eta_{Y}$, then $f \bar{u}=\overline{f u}$, that is, $f(u / F h)=(f u) / F h$.

Conversely, let $A$ be Kan-injective with respect to $F h$, consider a morphism $w: X \rightarrow G A$, and let $\bar{w}: F X \rightarrow A$ be the unique morphism such that $G \bar{w} \cdot \eta_{X}=w$. We show that $w / h=$ $G(\bar{w} / F h) \eta_{Y}$. Indeed, $G(\bar{w} / F h) \eta_{Y} h=G(\bar{w} / F h) \cdot G F h \cdot \eta_{X}=G \bar{w} \cdot \eta_{X}=w$; and, if $s: Y \rightarrow G A$ satisfies the inequality $w \leq s h$, then, for $s=G \bar{s} \cdot \eta_{Y}$, we have $G \bar{w} \cdot \eta_{X} \leq G \bar{s} \cdot \eta_{Y} \cdot h=G \bar{s}$. $G F h \cdot \eta_{X}=G(\bar{s} \cdot F h) \cdot \eta_{X}$, and, thus, $\bar{w} \leq \bar{s} F h$. It follows that $\bar{w} / F h \leq \bar{s}$, hence $G(\bar{w} / F h) \cdot \eta_{Y} \leq$ $G \bar{s} \cdot \eta_{Y}=s$. Again the property for morphisms easily follows.

Remark 2.9. We will also use the notion of Kan-projectivity, dual of the one of Kaninjectivity. Namely, in an order-enriched category $X$, an object $A$ is right Kan-projective with respect to a morphism $h: X \rightarrow Y$ if, for every morphism $f: A \rightarrow Y$ there is a (unique) morphism

$$
\frac{f}{h}: A \rightarrow X
$$

such that

(i) if $s: A \rightarrow X$ is a morphism with $f \geq h s$, then $\frac{f}{h} \geq s$;

(ii) $h \frac{f}{h}=f$.

And a morphism $g: A \rightarrow B$ is said to be right Kan-projective with respect to $h$ if the objects $A$ and $B$ are so, and $g$ satisfies the equality $\frac{f g}{h}=\frac{f}{h} g$ for every $f: B \rightarrow Y$.

Reversing the order we obtain left Kan-projectivity.

\section{3. n-FLAT EMBEDDINGS IN LOC}

We recall that a frame is a complete lattice satisfying the infinite distributive law

$$
a \wedge(\vee B)=\vee\{a \wedge b \mid b \in B\}
$$

for all its elements $a$ and subsets $B$. And a frame homomorphism between frames is a map preserving all joins and all finite meets. We denote the category of frames and frame homomorphisms by Frm. Its opposite category is the category Loc of locales and localic maps. Thus the objects of Loc, known as locales, are just the frames. The morphisms of Loc, the localic maps, may be seen as infima-preserving maps $f: L \rightarrow M$ such that the corresponding left-adjoint $f^{*}: M \rightarrow L$ preserves finite meets ([17], [25]). We recall that the fact that $f$ preserves arbitrary meets implies that it has a left adjoint defined by

$$
f^{*}(y)=\wedge\{x \in L \mid y \leq f(x)\}
$$


and $f^{*}$ preserves arbitrary joins. Analogously, every frame homomorphism $f$ has a right adjoint which preserves arbitrary meets.

Let us consider the categories Frm and Loc order-enriched via the pointwise order, that is, in both cases, for $f, g: X \rightarrow Y, f \leq g$ iff $f(x) \leq g(x), x \in X$. Then, for $f, g: X \rightarrow Y$ taken in Frm, we have that

$$
f \leq g \text { in Frm iff } f_{*} \geq g_{*} \text { in Loc }
$$

and, analogously, for $f, g: X \rightarrow Y$ taken in Loc,

$$
f \leq g \text { in Loc iff } f^{*} \geq g^{*} \text { in Frm. }
$$

Indeed, if $f$ and $g$ lie in Loc with $f(x) \leq g(x), x \in X$, then, for every $y \in Y$,

$$
g^{*}(y)=\wedge\{x \in X \mid y \leq g(x)\} \leq \wedge\{x \in X \mid y \leq f(x)\}=f^{*}(y) .
$$

Similarly, we conclude (3). As a consequence, an adjoint pair $f \dashv g$ in Frm gives rise to the adjoint pair $f_{*} \dashv g_{*}$ in Loc, and vice-versa.

It is easy to see that left Kan-injectivity in Loc translates into right Kan-projectivity in Frm. More precisely, a frame $A$ is right Kan-projective in Frm with respect to a frame homomorphism $h: X \rightarrow Y$ iff $A$ is left Kan-injective in Loc with respect to the localic map $h_{*}: Y \rightarrow X$. And the existence of $\frac{g}{h}$ for $g: A \rightarrow Y$ implies that $g_{*} / h_{*}$ exists in Loc and is given by the formula

$$
g_{*} / h_{*}=\left(\frac{g}{h}\right)_{*}
$$

Analogously, if $A$ is a locale left Kan-injective with respect to a localic map $h: X \rightarrow Y$, then $A$ is right Kan-projective with respect to $h^{*}$ in Frm and it holds the formula

$$
\frac{g^{*}}{h^{*}}=(g / h)^{*} \text {. }
$$

Terminology 3.1. From now on we proceed with just the terminologies Kan-projectivity in Frm and Kan-injectivity in Loc, referring, in the former case, to right Kan-projectivity, and, in the latter one, to left Kan-injectivity.

A localic map $f: L \rightarrow M$ is said to be an embedding if it is one-to-one. This is equivalent to its left-adjoint $f^{*}: M \rightarrow L$ being a surjection, and also equivalent to $f$ satisfying the equality $f^{*} f=i d_{L}$. Embeddings are precisely the extremal monomorphisms of Loc ([17], [25]).

Definition 3.2. For every cardinal $n \geq 1$, an embedding $g: L \rightarrow M$ in Loc is said to be $n$-flat provided that it preserves suprema of families of cardinality less or equal than $n$.

Remark 3.3. A 1-flat embedding is an embedding which preserves the least element, that is, a dense embedding. The 2-flat embeddings are the usual flat embeddings, that is, those embeddings which preserve finite joins (see [17]). 
Remark 3.4. The category Frm is a subcategory of the category SLat of meet-semilattices and homomorphisms, and the forgetful functor from Frm to SLat has a left adjoint

$$
D: \text { SLat } \rightarrow \text { Frm. }
$$

It sends every semilattice $L$ to the frame $D L$ of all lower sets of $L$ ordered by inclusion, that is, the elements of $D L$ are all subsets $W$ of $L$ with

$$
W=\downarrow W=\{x \in L \mid x \leq w \text { for some } w \in W\} ;
$$

and, given a morphism $f: L \rightarrow M$ of SLat, $(D f)(U)=\downarrow f[U]$ for all $U \in D L$. For every $L \in$ SLat, the corresponding universal morphism $d_{L}: L \rightarrow D L$ is defined by

$$
d_{L}(x)=\downarrow x=\{y \in L \mid y \leq x\}, x \in L ;
$$

and, given $f: L \rightarrow M$ in SLat with $M$ in Frm, the unique frame homomorphism $\bar{f}: D L \rightarrow$ $M$ with $\bar{f} d_{L}=f$ is defined by $\bar{f}(U)=\bigvee f[U]$. Moreover, Frm is a KZ-monadic subcategory of SLat via the reflector functor $D$. This follows from [4], and can also be easily shown by using (b) of Theorem 2.4. Indeed, (i) and (ii) are immediately seen; in addition, given a commutative square as in Definition 2.3, lying in SLat and with $f$ in Frm, it is easy to see that the semilattice $X$ has suprema given by the formula $\bigvee_{i \in I} a_{i}=x\left(\bigvee_{i \in I} x_{*}\left(a_{i}\right)\right)$, and analogously for $Y$; using this fact, it follows easily that Frm is closed under left adjoint retractions in SLat.

Also the forgetful functor from SLat to the category Set of sets and maps has a left adjoint $S:$ Set $\rightarrow$ SLat. It sends every set $X$ to the set of all finite subsets of $X$ equipped with the partial order given by $A \leq B$ iff $A \supseteq B$.

Consequently, given a set $X$, the free frame generated by $X$ may be constructed as follows $([17,25])$ :

First we form the free meet-semilattice $S X$ generated by $X$. The free frame generated by $X$, denoted $F X$, is precisely $D(S X)$. The corresponding universal map $\eta_{X}: X \rightarrow F X$ is then defined by

$$
\begin{aligned}
\eta_{X}(x)=\downarrow\{x\} & =\left\{W \in S X \mid W \leq_{S X}\{x\}\right\}=\{W \in S X \mid\{x\} \subseteq W\} \\
& =\{W \subseteq X \mid W \text { is finite and } x \in W\} .
\end{aligned}
$$

Notation 3.5. Let $n$ be any cardinal looked just as a set. In the following,

$$
F_{n}
$$

denotes the free frame generated by $n$. In particular, we put $F_{0}=(0<1)$ and $F_{1}=(0<d<$ $1)$.

Next we characterize the embeddings of Loc via Kan-injectivity.

Proposition 3.6. In Loc, a localic map $h$ is an embedding if and only if the three-element chain is Kan-injective with respect to $h$, if and only if every finite chain is Kan-injective with respect to $h$. 
Proof. Let $F_{1}=(0<d<1)$ be Kan-injective with respect to $h: X \rightarrow Y$ in Loc. We want to show that $h$ is one-to-one. For every $x \in X$, let $g_{x}: X \rightarrow F_{1}$ be the localic map given by

$$
g_{x}(z)= \begin{cases}1, & \text { if } z=1 \\ d, & \text { if } 0 \neq x \leq z \neq 1 \\ 0, & \text { otherwise }\end{cases}
$$

Then there exists the localic map $g_{x} / h: Y \rightarrow F_{1}$. For $x$ and $x^{\prime}$ in $X$ with $x \neq x^{\prime}$, assume, without loss of generality, that $x \not \leq x^{\prime}$. Then $g_{x}(x) \neq g_{x}\left(x^{\prime}\right)$ and, since $g_{x}=\left(g_{x} / h\right) h$, we conclude that $h(x) \neq h\left(x^{\prime}\right)$.

We prove now that every finite chain is Kan-injective with respect to all embeddings. We are going to work in the category Frm. Thus we want to show that finite chains are Kan-projective with respect to the frame homomorphisms $h: X \rightarrow Y$ for which $h_{*}: Y \rightarrow X$ satisfies the equality $h h_{*}=i d_{Y}$. Indeed, let $A$ be a finite chain and let $h: X \rightarrow Y$ be a surjection in Frm. Let $f: A \rightarrow Y$ be a morphism of Frm. We are going to show that the morphism $\frac{f}{h}: A \rightarrow X$ exists and is defined by:

$$
\frac{f}{h}(a)=\left(h_{*} f\right)(a) \text {, if } a \neq 0 \text {, and } \frac{f}{h}(0)=0 .
$$

We recall that the right adjoint of the morphism $h$ is given by $h_{*}(y)=\bigvee h^{-1}(\downarrow y)$, where $\downarrow y=\{z \in Y \mid z \leq y\}$. The function $\frac{f}{h}$ defined as above is monotonous, because

$$
\begin{aligned}
a \leq a^{\prime} & \Rightarrow \downarrow f(a) \subseteq \downarrow f\left(a^{\prime}\right) \\
& \Rightarrow \vee h^{-1}(\downarrow f(a)) \leq \vee h^{-1}\left(\downarrow f\left(a^{\prime}\right)\right) .
\end{aligned}
$$

Since $A$ is a finite chain, we conclude that $\frac{f}{h}$ preserves all the meets and non-empty joins; by definition it also preserves the empty joins. So it is a morphism of Frm. It is now easy to see that $\frac{f}{h}$ defined as in (7) satisfies (i) and (ii) of Remark 2.9.

Remark 3.7. Let $L$ be a meet-semilattice and let $D L$ be the frame of its lower sets ordered by inclusion (as described in Remark 3.4). Then, for every lower set $W \in D L$, we have that $W=\downarrow W=\bigcup_{a \in W} \downarrow a$, where $\downarrow a=\{b \in L \mid b \leq a\}$. Consider the set

$$
\bar{L}=\{\downarrow a \mid a \in L\} .
$$

Then every $W \in D L$ may be expressed as a supremum in $D L$ as follows: $W=\bigvee\{A \in$ $\bar{L} \mid A \leq W\}$. In particular, given a set $X$, the free frame generated by $X$ has the property that every $W \in F X$ can be expressed as a supremum of elements of $\overline{S X}$, where $S X$ is the meet-semilattice generated by $X$ and $\overline{S X}$ is defined as in (8):

$$
W=\bigvee\{A \in \overline{S X} \mid A \leq W\} .
$$

In order to point out another feature of the free frame $F X$, observe that, for every $x \in X,\{x\}$ is an element of the semilattice $S X$ and the element $\downarrow\{x\}$ of $F X$ is defined by $\downarrow\{x\}=\{A \in S X \mid x \in A\}$. The elements $\{x\}$ of $S X$ are clearly immediate predecessors of the 
greatest element $1_{S X}$, and, consequently, in the frame $F X$, the supremum $\bigvee_{x \in X} \downarrow\{x\}$ is an immediate predecessor of $1_{F X}$, indeed the only one.

Notation 3.8. For every cardinal $n$, we denote by $s$ the (unique) predecessor of $1_{F_{n}}$ :

$$
s=\bigvee_{k \in n} \downarrow\{k\} .
$$

In $F_{0}=(0<1)$ this element $s$ is just 0 , and in $F_{1}=(0<d<1)$ it is precisely $d$.

We are going to consider the localic map

$$
f_{n}: F_{n} \rightarrow F_{1}
$$

defined by $f_{n}(1)=1, f_{n}(s)=d$, and $f_{n}(x)=0$ for $x \neq 1$, s. In particular, $f_{0}(0)=d$ and $f_{1}$ is the identity map.

For every cardinal $n \geq 1$, let

$$
\mathcal{D}_{n}
$$

denote the category whose objects are $F_{0}, F_{1}$ and $F_{n}$, and whose morphisms, besides the identities, are $f_{0}$ and $f_{n}$. Of course, since $f_{1}$ coincides with the identity, $\mathcal{D}_{1}$ has just a non-identity morphism, which is $f_{0}$.

In Proposition 3.6 we characterized the embeddings of Loc in terms of Kan-injectivity of $F_{1}$. In the next proposition and theorem we characterize dense embeddings and $n$-flat embeddings by means of Kan-injectivity.

Proposition 3.9. Dense embeddings in Loc are precisely the localic maps with respect to which the morphism $f_{0}$ is Kan-injective.

Proof. From Proposition 3.6 we already know that the localic maps with respect to which $F_{0}$ and $F_{1}$ are Kan-injective are precisely the embeddings. Now we prove that an embedding $h: X \rightarrow Y$ is dense iff $f_{0}$ is Kan-injective with respect to it. We know that $f_{0}$ is Kan-injective with respect to $h$ in Loc iff $f_{0}^{*}$ is Kan-projective with respect to $h^{*}$ in Frm, that is, iff, for every frame homomorphism $g: F_{0} \rightarrow X$, the lower triangle of the diagram

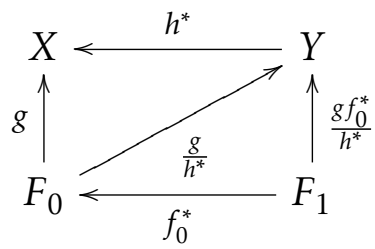

is commutative. Since we are dealing with frame homomorphisms, which preserve the least and greatest elements, we just need to prove that the equality

$$
\frac{g f_{0}^{*}}{h^{*}}(d)=\frac{g}{h^{*}} f_{0}^{*}(d)
$$

holds iff $h$ is dense, i.e., iff $h(0)=0$. Indeed, by using formula (7) of the proof of Proposition 3.6 for morphisms $f$ whose domain is a finite chain, and using the preservation of 
the least element by the frame homomorphisms $g$ and $\frac{g}{h^{*}}$, we have that:

$$
\frac{g f_{0}^{*}}{h^{*}}(d)=h g f_{0}^{*}(d)=h g\left(\bigwedge f_{0}^{-1}(\uparrow d)\right)=h(g(0))=h(0)
$$

and

$$
\frac{g}{h^{*}} f_{0}^{*}(d)=\frac{g}{h^{*}}(0)=0 .
$$

Then $f_{0}$ is Kan-injective with respect to $h$ if and only if the right-hand sides of the equations (11) and (12) are equal, that is, iff $h(0)=0$.

Theorem 3.10. For every cardinal $n \geq 1, n$-flat embeddings in Loc are precisely the localic maps with respect to which $f_{0}$ and $f_{n}$ are Kan-injective.

Proof. In order to show that $F_{n}$ is Kan-injective with respect to $n$-flat embeddings, we work in Frm: we prove that $F_{n}$ is Kan-projective with respect to frame homomorphisms $h: X \rightarrow Y$ whose right adjoint $h_{*}$ fulfils $h h_{*}=\mathrm{id}_{Y}$ and preserves unions of families indexed by $k \leq n$.

Let then $h: X \rightarrow Y$ be of that form, and let $g: F_{n} \rightarrow Y$ be a frame homomorphism. We show that $h_{*} g$ is a frame homomorphism. (Moreover, it will be clear from the proof that the same is true for any $g: F_{i} \rightarrow Y$ with $i<n$.) This will guarantee that

$$
\frac{g}{h}=h_{*} g \text {, }
$$

since we have that $h\left(h_{*} g\right)=g$, and $h s \leq g \Rightarrow h_{*} h s \leq h_{*} g \Rightarrow s \leq h_{*} g$.

In order to show that $h_{*} g$ is indeed a frame homomorphism, we observe that it preserves finite meets because $h_{*}$ is a right adjoint and $g$ is a frame homomorphism. And $h_{*} g$ preserves the least element because $h_{*}$ is dense. Moreover, by hypothesis, $h_{*} g$ preserves suprema of every set of cardinality no greater than $n$. We are to prove that the same is true for arbitrary suprema. Let then $\left\{W_{t}: t \in T\right\}$ be a subset of $F_{n}$, and let us consider the subset of $F_{n}$ given by $\overline{S n}=\{\downarrow a \mid a \in S n\}$, which is defined by departing from the semilattice $S n$ accordingly to formula (8) of Remark 3.7. Following formula (9), we know that, for every $t \in T, W_{t}=\bigvee\left\{A \in \overline{S n}: A \leq W_{t}\right\}$. Then, we have that

$$
\begin{aligned}
\left(h_{*} g\right)\left(\bigvee_{t \in T} W_{t}\right) & =\left(h_{*} g\right)\left(\bigvee_{t \in T}\left(\bigvee\left\{A \in \overline{S n}: A \leq W_{t}\right\}\right)\right) \\
& =\left(h_{*} g\right)\left(\bigvee\left\{A \in \overline{S n}: A \leq W_{t} \text { for some } t \in T\right\}\right) .
\end{aligned}
$$

The cardinality of $\overline{S n}$, which is equal to the one of $S n$, is finite if $n$ is so, and it is equal to $n$ if $n$ is infinite. Consequently, we know that $h_{*}$ preserves suprema of $g[M]$, for every 
$M \subseteq \overline{S n}$. Therefore, using also the fact that $g$ is a morphism of Frm, we have:

$$
\begin{aligned}
\left(h_{*} g\right)\left(\bigvee_{t \in T} W_{t}\right) & =h_{*}\left(\bigvee\left\{g(A): A \in \overline{S n} \text { and } A \leq W_{t} \text { for some } t \in T\right\}\right) \\
& =\bigvee\left\{\left(h_{*} g\right)(A): A \in \overline{S n} \text { and } A \leq W_{t} \text { for some } t \in T\right\} \\
& =\bigvee\left(\bigcup_{t \in T}\left\{\left(h_{*} g\right)(A): A \in \overline{S n} \text { and } A \leq W_{t}\right\}\right) \\
& =\bigvee_{t \in T}\left(\bigvee_{\{}\left\{\left(h_{*} g\right)(A): A \in \overline{S n} \text { and } A \leq W_{t}\right\}\right) \\
& =\bigvee_{t \in T}\left(h_{*} g\right)\left(\bigvee\left\{A \in \overline{S n}: A \leq W_{t}\right\}\right) \\
& =\bigvee_{t \in T}\left(h_{*} g\right)\left(W_{t}\right) .
\end{aligned}
$$

Consequently, given an $n$-flat embedding $m: X \rightarrow Y$ in Loc, and a localic map $l: X \rightarrow F_{i}$ with $i \leq n$, using the formula (5) and the equality (13), we have

$$
l / m=\left(\frac{l^{*}}{m^{*}}\right)_{*}=\left(\left(m^{*}\right)_{*} l^{*}\right)_{*}=\left(m l^{*}\right)_{*}=l m_{*}
$$

with $l m_{*}$ a localic map. In order to verify that $f_{n}$ is Kan-injective with respect to $m: X \rightarrow$ $Y$, it suffices now to use (14) applied to $l$ and $f_{n} l$ :

$$
f_{n}(l / m)=f_{n}\left(l m_{*}\right)=\left(f_{n} l\right) m_{*}=\left(f_{n} l\right) / m .
$$

Conversely, let $f_{n}$ be Kan-injective with respect to the embedding $m: X \rightarrow Y$ in Loc; equivalently, $f_{n}^{*}$ is Kan-projective with respect to $m^{*}: Y \rightarrow X$ in Frm. We already know from the above proposition that $m$ preserves the supremum of the empty family. We want to prove that $m$ preserves suprema of non-empty $n$-indexed families. For that, let $\left\{x_{k} \mid k \in n\right\}$ be a set of elements of $X$. Define a map $p: n \rightarrow X$ by putting $p(k)=x_{k}$. Let $\eta_{n}: n \rightarrow F_{n}$ be the universal map given by $\eta_{n}(k)=\downarrow\{k\}$ (see Remark 3.4). Then there is a unique frame homomorphism $g: F_{n} \rightarrow X$ such that $g \eta_{n}=p$; in particular, $g(\downarrow\{k\})=x_{k}$ for every $k \in n$, and then, $g(s)=g\left(\bigvee_{k \in n} \downarrow\{k\}\right)=\bigvee_{k \in n} x_{k}$.

By hypothesis, there exist the morphisms $\frac{g}{m^{*}}$ and $\frac{g f_{n}^{*}}{m^{*}}$ in Frm, and, moreover, they satisfy the equality

In particular,

$$
\frac{g f_{n}^{*}}{m^{*}}=\frac{g}{m^{*}} f_{n}^{*}
$$

$$
\frac{g f_{n}^{*}}{m^{*}}(d)=\frac{g}{m^{*}} f_{n}^{*}(d)
$$


On one hand, taking into account that $f_{n}^{*}(d)=\bigwedge\left\{z \in F_{n} \mid d \leq f_{n}(z)\right\}=s$, and the formula (7) obtained in the proof of Proposition 3.6, we have:

$$
\frac{g f_{n}^{*}}{m^{*}}(d)=m g f_{n}^{*}(d)=m g(s)=m\left(\bigvee_{k \in n} x_{k}\right)
$$

Since $\mathrm{id}_{Y} \leq m m^{*}$ and $m^{*} \frac{g}{m^{*}}=g$, then $\frac{g}{m^{*}} \leq m m^{*} \frac{g}{m^{*}}=m g$. Thus, using also the fact that $\frac{g}{m^{*}}$ is a frame homomorphism, we obtain, on the other hand, that

$$
\frac{g}{m^{*}} f_{n}^{*}(d)=\frac{g}{m^{*}}(s)=\frac{g}{m^{*}}\left(\bigvee_{k \in n} \downarrow\{k\}\right)=\bigvee_{k \in n} \frac{g}{m^{*}}(\downarrow\{k\}) \leq \bigvee_{k \in n} m(g(\downarrow\{k\}))=\bigvee_{k \in n} m\left(x_{k}\right) .
$$

From (15), (16) and (17), we obtain $m\left(\bigvee_{k \in n} x_{k}\right) \leq \bigvee_{k \in n} m\left(x_{k}\right)$. As the other inequality trivially holds, we conclude that $m\left(\bigvee_{k \in n} x_{k}\right)=\bigvee_{k \in n} m\left(x_{k}\right)$.

Remark 3.11. In the proof of the above theorem we saw that the locale $F_{n}$ is Kan-injective with respect to $n$-flat embeddings. More than that, it is Kan-injective with respect to all embeddings. In other words, every free frame is Kan-projective with respect to quotients in Frm. Indeed, let $D:$ SLat $\rightarrow$ Frm and $S:$ Set $\rightarrow$ SLat be the functors described in Remark 3.4. Given a quotient $h: X \rightarrow Y$ in Frm and a frame homomorphism $g: F_{n} \rightarrow Y$, it is easy to see that the morphism $\frac{g}{h}$ is the frame homomorphism $\hat{g}: F_{n}=D S n \rightarrow X$ with $h_{*} g d_{S n}=\hat{g} d_{S n}$, where $d_{S n}$ is the universal morphism from $S n$ to Frm. Indeed, the equality $h \hat{g}=g$ follows from the equalities $h \hat{g} d_{S n}=h h_{*} g d_{S n}=g d_{S_{n}}$, and, given a frame homomorphism $s: F_{n} \rightarrow X$ with $h s \leq g$, then we have $s d_{S n} \leq h_{*} h s d_{S n} \leq h_{*} g d_{S n}=\hat{g} d_{S n}$. Thus, since the reflection is of KZ type, as observed in Remark 3.4, it follows that $s \leq \hat{g}$ (see Theorem 2.4 and Remark 2.5).

Remark 3.12. It is well known that there is a contravariant functor $\Omega$ from Top to Frm sending every space $X$ to the frame $\Omega X$ of its open subsets and every continuous map $f: X \rightarrow Y$ to the frame homomorphism $f^{-1}: \Omega Y \rightarrow \Omega X$. This functor, seen as a covariant one, may be defined as the functor

$$
\text { Lc : Top } \rightarrow \text { Loc }
$$

sending every continuous map $f: X \rightarrow Y$ to the right-adjoint $\left(f^{-1}\right)_{*}: \Omega X \rightarrow \Omega Y$ of $f^{-1}$ : $\Omega Y \rightarrow \Omega X$, thus $\left(f^{-1}\right)_{*}(H)=\bigcup\left\{V \in \Omega Y: f^{-1}(V) \subseteq H\right\}, H \in \Omega X$. Moreover, a locale is said to be spatial if it is isomorphic to the frame $\Omega X$ for some space $X$.

The union of all subcategories $\mathcal{D}_{n}$ defined in Notation 3.8 is a subcategory

of Loc. The category $\mathcal{D}$ is made of spatial locales, since the lower sets of a meet-semilattice $L$ form a topology over the underlying set of $L$. Furthermore, as a consequence of Theorem 3.10, we have the following: 
Corollary 3.13. The Kan-injective hull of $\mathcal{D}$ in Loc is the entire category Loc.

Proof. Let us denote by $\mathbb{E}_{n}$ the class of all $n$-flat embeddings, and by $\mathbb{E}_{C}$ the class of all completely flat embeddings, that is, embeddings that preserve arbitrary suprema. Then, using the fact that the operators _ KInj and KInj(-) establish a Galois connection between subcategories and classes of morphisms, we have:

$$
\mathcal{D}^{\mathrm{KInj}}=\left(\bigcup_{n \geq 1} \mathcal{D}_{n}\right)^{\mathrm{KInj}}=\bigcap_{n \geq 1} \mathcal{D}_{n}^{\mathrm{KInj}}=\bigcap_{n \geq 1} \mathbb{E}_{n}=\mathbb{E}_{C} .
$$

A completely flat morphism $f: X \rightarrow Y$ of Loc is a frame homomorphism, thus $f_{*}$ also belongs to Loc. As a consequence, it follows that, if $f$ is, moreover, an embedding, that is, $f_{*} f=\mathrm{id}$, then all locales and all localic maps are Kan-injective with respect to $f$ : for every $g: X \rightarrow A$ in Loc, $g / f=g f_{*}$. Therefore,

$$
\mathbf{K}(\mathcal{D})=\operatorname{KInj}\left(\mathcal{D}^{\mathrm{KInj}}\right)=\operatorname{KInj}\left(\mathbb{E}_{C}\right)=\text { Loc. }
$$

\section{The CATEgories $\operatorname{Loc}_{n}$}

In this section we study the Kan-injective hulls in Loc of the subcategories $\mathcal{D}_{n}$ of the last section. In particular, we will see that the Kan-injective hull of $\mathcal{D}_{n}$ is precisely the Eilenberg-Moore category of a KZ-monad $G_{n}$ : Loc $\rightarrow$ Loc. For that, we are going to use some results of [4], as explained in the following remark.

Remark 4.1. Let $D:$ SLat $\rightarrow$ Frm be the reflector functor already described in Remark 3.4.

1. Banaschewski [4] considered subcategories $\mathcal{K}$ of the category SLat obtained as follows: Let

$$
(S A)_{A \in \mathrm{SLat}}
$$

be a family where, for every object $A, S A$ is a subset of the power-set of $A$, satisfying the following conditions:

(a) $\{a \wedge t \mid t \in P\} \in S A$, for every $a \in A$ and every $P \in S A$.

(b) for every morphism $f: A \rightarrow B$ of SLat and every $P \in S A, f[P] \in S B$.

The corresponding category

$$
\mathcal{K}
$$

is then constituted by the objects $A$ of SLat such that for every $P \in S A, \vee P$ exists and $a \wedge(\vee P)=\vee\{a \wedge t \mid t \in P\}$, and by the morphisms $f: A \rightarrow B$ of SLat such that $f(\vee P)=\vee f[P]$, $P \in S A$.

For a subcategory $\mathcal{K}$ obtained this way, Banaschewski proved that Frm is reflective in $\mathcal{K}$ with every reflection $\eta_{A}: A \rightarrow R A$ being given by

$$
R A=\{U \in D A \mid(P \subseteq U \text { and } P \in S A) \Rightarrow \vee P \in U\} \text {, and } \eta_{A}(a)=\downarrow a, a \in A .
$$


For every morphism $f: A \rightarrow L$ in $\mathcal{K}$, with $L \in$ Frm, the unique morphism $\bar{f}: R A \rightarrow L$ such that $\bar{f} \eta_{A}=f$ is defined by $\bar{f}(U)=\vee f[U]$. The counit is thus given by $\varepsilon_{L}(U)=\vee U, U \in R L$.

This reflection induces then a comonad in Frm whose functor part is the composition of the inclusion of Frm into SLat with the reflector functor $R$, and this comonad is of KZ type ([4]).

2. For every cardinal $n$, let

$$
\left(S_{n} A\right)_{A \in \mathrm{SLat}}
$$

be the family defined by:

$$
\begin{aligned}
& S_{0} A=\emptyset ; \text { and } \\
& S_{n} A=\{P \subseteq A \mid \operatorname{card}(P) \leq n\}, \text { for } n \geq 1 .
\end{aligned}
$$

It is easy to see that $S_{n}$ satisfies the conditions described for $S$ above. Let us denote the corresponding subcategory $\mathcal{K}$ and its reflector in Frm by $\mathcal{K}_{n}$ and $R_{n}$, respectively. For $n=0, \mathcal{K}_{0}$ is just SLat, and, for $n \geq 1$, it consists of all objects $A$ and morphisms $f: A \rightarrow B$ of SLat such that, for all $a \in A$ and $P \subseteq A$ with $\operatorname{card}(P) \leq n, \vee P$ exists, $a \wedge(\vee P)=\bigvee\{a \wedge t \mid t \in P\}$, and $f[\vee P]=\vee f[P]$. The comonad over Frm induced by the reflector

$$
R_{n}: \mathcal{K}_{n} \rightarrow \text { Frm }
$$

is precisely $\mathbf{H}_{\mathbf{n}}=\left(H_{n}, \varepsilon, \delta\right)$, defined as follows:

The functor

$$
H_{n}: \text { Frm } \rightarrow \text { Frm }
$$

sends each frame $L$ to the frame

$$
H_{n} L=\{U \in D L \mid(P \subseteq U, \operatorname{card}(P) \leq n) \Rightarrow \vee P \in U\}
$$

ordered by inclusion. Moreover, for each frame homomorphism $f: L \rightarrow M, H_{n} f: H_{n} L \rightarrow$ $H_{n} M$ is defined by

$$
H_{n} f(U)=\bigvee\{\downarrow f(u) \mid u \in U\}, U \in H_{n} L .
$$

The counit $\varepsilon$ is given, for every $L \in$ Frm and $U \in H_{n} L$, by

$$
\varepsilon_{L}(U)=\vee U .
$$

The comultiplication $\delta: H_{n} \rightarrow H_{n} H_{n}$ is given, for every $L \in \operatorname{Frm}$ and $U \in H_{n} U$, by $\delta_{L}(U)=$ $\bigvee\left\{\downarrow_{H_{n} L}\left(\downarrow_{L} u\right) \mid u \in U\right\}$.

Moreover, this comonad is of $\mathrm{KZ}$ type, more precisely, it satisfies the inequalities

$$
\varepsilon_{H_{n} X} \leq H_{n} \varepsilon_{X}, X \in \text { Frm. }
$$

Notation 4.2. 1. By duality, the comonad $\mathbf{H}_{\mathbf{n}}$ yields a monad in Loc; let

$$
G_{n}: \text { Loc } \rightarrow \text { Loc }
$$


be its corresponding functor; thus, for every localic map $f, G_{n} f=\left(H_{n} f^{*}\right)_{*}$. The unit of the monad $\mathbf{G}_{n}$ over Loc is given by $\varepsilon_{X}^{\text {op }}=\left(\varepsilon_{X}\right)_{*}$. As a consequence of the inequalities (18), taking into account that the order of the morphisms is reversed in the passage from Frm to Loc, we obtain the inequalities

$$
\varepsilon_{H_{n} X}^{\mathrm{op}} \geq H_{n}^{\mathrm{op}} \varepsilon_{X}^{\mathrm{op}}
$$

in Loc. These inequalities mean that this monad is of $\mathrm{KZ}$ type (see 2.2.) Accordingly, our $G_{n}$-embeddings are going to be those localic maps $f: X \rightarrow Y$ such that $G_{n} f$ is a left adjoint section, i.e., it has a right adjoint $\left(G_{n} f\right)_{*}$ in Loc with $\left(G_{n} f\right)_{*} G_{n} f=\mathrm{id}_{G_{n} X}$ (see Proposition 2.7).

2. Aiming to give a characterization of the subcategories $\operatorname{KInj}\left(\mathbb{E}_{n}\right)$ of Loc for $\mathbb{E}_{0}$ the class of embeddings and $\mathbb{E}_{n}$ (with $n \geq 1$ ) the class of $n$-flat embeddings, let us consider, for every cardinal $n$ and every frame $L$, the binary relation defined in the underlying set of $L$ as follows:

$$
x \triangleleft_{n} a \Leftrightarrow\left(\forall U \in H_{n} L, a \leq \vee U \Rightarrow x \in U\right) .
$$

This kind of relation was possibly used for the first time by Raney in [26], and Banaschewski [4] considered a generalization of it which encompasses all comonads induced by a reflector $R: \mathcal{K} \rightarrow$ Frm of the type mentioned in Remark 4.1.

We denote by

$$
\operatorname{Loc}_{n}
$$

the subcategory of Loc whose objects are the locales $L$ that satisfy the following conditions:

(i) For every $a \in L, a=\bigvee_{x \triangleleft_{n} a} x$,

(ii) Given $x, y, a \in L$, if $x \triangleleft_{n} a$ and $x \triangleleft_{n} b$ then $x \triangleleft_{n} a \wedge b$,

(iii) $1_{L} \triangleleft_{n} 1_{L}$;

and whose morphisms are all the localic maps $f$ with a left adjoint $f^{*}$ that preserves the relation " $\triangleleft_{n}$ ".

Remark 4.3. The objects of the category $\operatorname{Loc}_{2}$ are precisely the stably locally compact locales and, moreover, they are spatial and coincide with the (left adjoint) retracts of coherent locales ([16], [18]). A localic map between coherent locales belongs to Loc $_{2}$ iff it is a coherent morphism in the sense of [17]. From Theorem 2.4, it follows that $\operatorname{Loc}_{2}$ is precisely the closure under left adjoint retractions, on objects and on morphisms, of the category of coherent locales and coherent localic maps. Furthermore, by Theorem 4.5 below and Proposition 2.7, the flat embeddings coincide with $\operatorname{Loc}_{2}{ }^{K I n j}$, that is, they form the largest class of localic maps with respect to which $\operatorname{Loc}_{2}$ is Kan-injective.

Remark 4.4. 1. From general properties of the reflector $R: \mathcal{K} \rightarrow$ Frm of Remark 4.1 proved in [4], we know that the Eilenberg-Moore coalgebras of the KZ-comonad $\mathbf{H}_{n}$ are precisely those frames which satisfy the three conditions (i)-(iii) of Notation 4.2 .2 , and 
also precisely those frames which are Kan-projective with respect to $n$-flat surjections (that is, with respect to the frame homomorphisms $f$ whose right adjoint $f_{*}$ is an $n$-flat embedding in Loc). In other words, a locale is Kan-injective with respect to the $n$-flat embeddings iff it is an object of Loc $_{n}$. Indeed Kan-injectivity of locales with respect to $n$-flat embeddings coincides, in the dual context, with $\mathcal{K}_{n}$-flat projectivity of frames in the sense of [4], where $\mathcal{K}_{n}$ is defined as in Remark 4.1.2.

In the next theorem we complete the characterization of the Eilenberg-Moore category of the monad $\mathbf{G}_{n}$ over Loc by describing also its morphisms.

2. We observe that, as it follows from Remark 2.2, by duality, the structure map of an Eilenberg-Moore coalgebra $(L, l)$ of the comonad $\mathbf{H}_{n}$ is left adjoint to $\varepsilon_{L}$, where $\varepsilon$ is the counit of the comonad $\mathbf{H}_{n}$; thus we have that $l \varepsilon_{L} \leq \mathrm{id}_{H_{n} L}$ and $\varepsilon_{L} l=\mathrm{id}_{L}$. Moreover, as it was proved in [4], $l$ is defined by

$$
l(a)=\bigvee_{x \triangleleft_{n} a} \downarrow x, a \in L,
$$

and the binary relation $\triangleleft_{n}$ is characterized by

$$
a \triangleleft_{n} b \Leftrightarrow \downarrow a \subseteq l(b), a, b \in L .
$$

Theorem 4.5. The Eilenberg-Moore category of the KZ-monad $G_{n}$ is $\operatorname{Loc}_{n}$. Moreover, $\operatorname{Loc}_{0}$ is the Kan-injective hull of $F_{1}$, and $\operatorname{Loc}_{n}$ is the Kan-injective hull of $\mathcal{D}_{n}$, for $n \geq 1$.

Proof. We already know from Notation 4.2 that $G_{n}$ yields a KZ-monad. Next we verify that the embeddings are precisely the $G_{0}$-embedings and, for $n \geq 1, n$-flat embeddings are precisely the $G_{n}$-embeddings (see Definition 2.3.2 and Proposition 2.7).

If $f: X \rightarrow Y$ is an embedding in Loc then it is a morphism of $\mathcal{K}_{0}=$ SLat (see Remark 4.1.2) such that the frame homomorphism $f^{*}$ satisfies the equality $f^{*} f=\mathrm{id}_{X}$. If, moreover, $f$ is $n$-flat, $f$ is a morphism of $\mathcal{K}_{n}$. The functor $R_{n}: \mathcal{K}_{n} \rightarrow$ Frm is locally monotone, then it preserves adjoint pairs and left inverses, that is, $R_{n} f^{*} \dashv R_{n} f$ in Frm and $R_{n} f^{*} R_{n} f=\mathrm{id}$. This implies that $\left(R_{n} f^{*}\right)_{*} \dashv\left(R_{n} f\right)_{*}$ in Loc and $\left(R_{n} f\right)_{*}\left(R_{n} f^{*}\right)_{*}=\mathrm{id}_{G_{n} X}$; equivalently, taking into account that $G_{n} f=\left(H_{n} f^{*}\right)_{*}=\left(R_{n} f^{*}\right)_{*}$, we have that $G_{n} f \dashv\left(R_{n} f\right)_{*}$ in Loc and $\left(R_{n} f\right)_{*} G_{n} f=\operatorname{id}_{G_{n} X}$, that is, $f$ is a $G_{n}$-embedding.

Conversely, assume that $f: X \rightarrow Y$ is a localic map such that $\left(G_{n} f\right)_{*} \in \operatorname{Loc}$ and $\left(G_{n} f\right)_{*} G_{n} f=$ $\operatorname{id}_{G_{n} X}$. Then we conclude that the map $\left(R_{n} f^{*}\right)_{*}$, which coincides with $G_{n} f$, lies in $\mathcal{K}_{n}$ and satisfies the equality $R_{n} f^{*}\left(R_{n} f^{*}\right)_{*}=\mathrm{id}_{R_{n} X}$. Let $\eta$ and $\varepsilon$ be the unit and counit relative to the reflector functor $R_{n}: \operatorname{Frm} \rightarrow \mathcal{K}_{n}$. Then, we have, on one hand,

$$
f^{*}\left(\varepsilon_{Y}\left(\left(G_{n} f\right) \eta_{X}\right)=f^{*}\left(\varepsilon_{Y}\left(R_{n} f^{*}\right)_{*} \eta_{X}\right)=\varepsilon_{X}\left(R_{n} f^{*}\right)\left(R_{n} f^{*}\right)_{*} \eta_{X}=\varepsilon_{X} \eta_{X}=\mathrm{id}{ }_{X} ;\right.
$$

and, on the other hand,

$$
\left(\varepsilon_{Y}\left(\left(G_{n} f\right) \eta_{X}\right) f^{*}=\left(\varepsilon_{Y}\left(R_{n} f^{*}\right)_{*} \eta_{X}\right) f^{*}=\varepsilon_{Y}\left(R_{n} f^{*}\right)_{*} R_{n} f^{*} \eta_{Y} \geq \varepsilon_{Y} \eta_{Y}=\mathrm{id}_{Y} .\right.
$$


We conclude that $f^{*} \dashv \varepsilon_{Y}\left(G_{n} f\right) \eta_{X}$, that is, $f=\varepsilon_{Y}\left(G_{n} f\right) \eta_{X}$; since the three morphisms of the last composition belong to $\mathcal{K}_{n}$, so do $f$, thus it preserves unions of $k$-indexed families, for $k \leq n$, i.e., $f$ is $n$-flat. The equality (21) assures that $f^{*} f=\mathrm{id}_{X}$, that is, $f$ is an embedding.

In order to complete the proof of the theorem, we have to show that the category $\operatorname{Loc}_{n}$ is the Eilenberg-Moore category of the monad $\mathbf{G}_{n}$. For objects the assertion immediately follows from [4], as we have said in Remark 4.4. It remains to prove that the morphisms of $\mathrm{Loc}_{n}$ are precisely the localic maps $f$ such that $f^{*}$ preserves the binary relation $\triangleleft_{n}$. Equivalently, we show that the morphisms of the category of the Eilenberg-Moore coalgebras of the comonad $\mathbf{H}_{n}$ are the frame homomorphisms that preserve the relation $\triangleleft_{n}$.

Let $(L, l)$ and $(M, m)$ be two coalgebras and let $f: L \rightarrow M$ be a morphism of coalgebras, i.e., we have the equality $\left(H_{n} f\right) l=m f$. To conclude that $f$ preserves the relation “ $\triangleleft_{n}$ ", let $a, b \in L$ be such that $a \triangleleft_{n} b$. Then we have:

$$
\begin{aligned}
a \triangleleft_{n} b & \Rightarrow \downarrow a \subseteq l(b) & & \text { by }(20) \\
& \Rightarrow\left(H_{n} f\right)(\downarrow a) \subseteq\left(H_{n} f\right)(l(b)) & & \text { because } H_{n} f \text { is a frame homomorphism } \\
& \Rightarrow \downarrow f(a) \subseteq m f(b) & & \text { since }\left(H_{n} f\right)(\downarrow a)=\bigvee\{\downarrow f(z) \mid z \leq a\}=\downarrow f(a) \\
& \Rightarrow f(a) \triangleleft_{n} f(b) & & \text { by }(20) .
\end{aligned}
$$

Conversely, suppose that $f: L \rightarrow M$ is a frame homomorphism whose right adjoint $f_{*}$ is a morphism of $\operatorname{Loc}_{n}$. Then there are morphisms $l$ and $m$ such that $(L, l)$ and $(M, m)$ are coalgebras; we want to show that $\left(H_{n} f\right) l=m f$. We know that $\varepsilon_{L} l=\mathrm{id}$ and $m \varepsilon_{M} \leq \mathrm{id}$ (see Remark 4.4.2); moreover, since $\varepsilon$ is a natural transformation, $\varepsilon_{M} H_{n} f=f \varepsilon_{L}$. Thus, we have that

$$
m f=m f \varepsilon_{L} l=m \varepsilon_{M}\left(H_{n} f\right) l \leq\left(H_{n} f\right) l .
$$

To show that also $\left(H_{n} f\right) l \leq m f$, let $a \in L$. Then, we have that

$$
\begin{aligned}
\left(H_{n} f\right)(l(a)) & =\left(H_{n} f\right)\left(\bigvee_{x \triangleleft_{n} a} \downarrow x\right), \quad \text { by }(19) \\
& =\bigvee_{x \triangleleft_{n} a}\left(H_{n} f\right)(\downarrow x), \quad \text { because } H_{n} f \text { is a frame homomorphism } \\
& =\bigvee_{x \triangleleft_{n} a} \downarrow f(x) .
\end{aligned}
$$

For $x \in L$ satisfying $x \triangleleft_{n} a$, we have $f(x) \triangleleft_{n} f(a)$, because $f$ preserves the relation $\triangleleft_{n}$; thus, $\left(H_{n} f\right)(l(a)) \leq \bigvee_{f(x) \triangleleft_{n} f(a)} \downarrow f(x) \leq \bigvee_{y \triangleleft_{n} f(a)} \downarrow y$, that is, taking into account the characterization of the coalgebra structure maps given in (19), $\left(H_{n} f\right)(l(a)) \leq m(f(a))$ in $H_{n} M$.

Remark 4.6. From the above theorem it follows that Loc is the union of an increasing chain of KZ-monadic subcategories that are Kan-injective hulls of finite subcategories. Indeed, we know that $\operatorname{Loc}_{n}=\operatorname{KInj}\left(\mathbb{E}_{n}\right)$, where, for every $n \geq 1, \mathbb{E}_{n}$ is the class of $n$-flat embeddings, and $\mathbb{E}_{0}$ is the class of embeddings; since $m \leq n$ clearly implies $E_{m} \supseteq \mathbb{E}_{n}$, it follows that $\operatorname{Loc}_{m}=\operatorname{KInj}\left(\mathbb{E}_{m}\right) \subseteq \operatorname{KInj}\left(\mathbb{E}_{n}\right)=\operatorname{Loc}_{n}$.

Recall that a cardinal $n$ is said to be regular if it cannot be expressed as the union of a family of sets of cardinality less than $n$ indexed by a set of cardinality also less then $n$. 
Thus the only finite regular cardinals are 0,1 and 2. If $n$ and $m$ are cardinals with $m<n$ and $n$ regular, then the category Loc $m$ is strictly contained in the category $\operatorname{Loc}_{n}$. Indeed, taking into account that, by Theorems 4.5 and 2.4, $\mathbb{E}_{n}=\operatorname{Loc}_{n} \mathrm{KInj}$, it suffices to show that $\mathbb{E}_{m} \neq \mathbb{E}_{n}$. For $m=0<1$, take the non dense embedding from the chain $(0<1)$ to the chain $(0<d<1)$ which sends 0 to $d$ and 1 to 1 . For $m=1<2$, take the embedding $h: A \rightarrow B$ where $A$ is the diamond consisting of the elements $a$ and $b$, its meet $a \wedge b=0$ and its join $a \vee b=1, B$ is the five-element frame with elements $a, b$, the meet $a \wedge b=0$, the join $a \vee b=s$ and the greatest element 1 , and $h$ is defined by $h(x)=x$ for all $x \in A$. Thus $h$ is dense but not flat. For $m<n$ with $n$ an infinite regular cardinal, let $h: n+1 \rightarrow n+2$ be the embedding from $n+1=\{0,1, \ldots, n\}$ to $n+2=\{0,1, \ldots, n, n+1\}$, both equipped with the usual order, defined by $h(k)=k$ if $k<n$ and $h(n)=n+1$. Then $h$ is clearly $m$-flat but it is not $n$-flat, because $h\left(\bigvee_{k \in n} k\right)=h(n)=n+1$ but $\bigvee_{k \in n} h(k)=\bigvee_{k \in n} k=n$.

\section{Kan-InJectivity in Top 0}

The study of full reflective subcategories, and the reflective hull of full subcategories, in categories of topological spaces, and in particular in the category Top $\mathrm{T}_{0}$, is a lively realm of research, see for instance, $[24,13,15,23,29]$. In the last section we have just seen that Loc is the union of an increasing chain of $\mathrm{KZ}$-monadic subcategories which are Kan-injective hulls, and then also KZ-monadic hulls, of finite subcategories. In this section we will use the results on Loc to show that an analogous situation occurs in the full subcategory Sob of $\mathrm{Top}_{0}$ of all sober spaces.

We recall that the specialization order is the partial order between the points of a $T_{0}$ topological space defined by $x \leq y$ iff every open set which contains $x$ also contains $y$. We are going to regard the category $\mathrm{Top}_{0}$ as an order-enriched category by considering the dual of this order pointwisely: given morphisms $f, g: X \rightarrow Y$, we put $f \leq g$ if $f^{-1}(G) \supseteq$ $g^{-1}(G)$ for all open sets $G$ of $Y$.

In the following, when we speak of Kan-injectivity in Top ${ }_{0}$, we refer to left Kan-injectivity.

Example 5.1. Let $f: X \rightarrow Y$ be an embedding in $\mathrm{Top}_{0}$ and let $S$ denote the Sierpiński space. For every open set $U$ of $X$, and $\chi_{U}: X \rightarrow S$ the characteristic map, we have that $\chi_{U} / f=\chi_{\left(f^{-1}\right)_{*}(U)}([5])$.

\section{Remark 5.2. Let}

$$
\text { Lc : } \mathrm{Top}_{0} \rightarrow \text { Loc }
$$

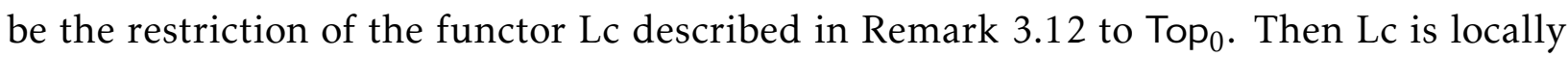
monotone, because $f \leq g$ in $\operatorname{Top}_{0}$ implies that $f^{-1} \geq g^{-1}$ in Frm, so $\left(f^{-1}\right)_{*} \leq\left(g^{-1}\right)_{*}$, that is, $\mathrm{Lc}(f) \leq \mathrm{Lc}(g)$ in Loc. We also recall that its right adjoint

$$
\Sigma: \text { Loc } \rightarrow \text { Top }_{0}
$$

sends every locale $A$ to the topological space $\sum A$ consisting of the pair $(\operatorname{pt}(A), \varphi)$, where $\operatorname{pt}(A)$ is the set of all frame homomorphisms $p: A \rightarrow \operatorname{Lc}(1) \cong F_{0}$ and $\varphi$ is the topology 
formed by the sets $\varphi(a)=\{p \in \operatorname{pt}(A) \mid p(a)=1\}$. The functor $\Sigma$ is also locally monotone as we show next. For that, recall that the counit $\varepsilon$ of the adjunction Lc $\dashv \sum$ behaves as follows: for every $f: \operatorname{Lc}(X) \rightarrow A$, the unique morphism $\bar{f}: X \rightarrow \sum A$ of Top $_{0}$ with $\varepsilon_{A} \operatorname{Lc}(\bar{f})=f$ is given by $\bar{f}(x)=\left(A \stackrel{f_{*}}{\longrightarrow} \operatorname{Lc}(X) \stackrel{\operatorname{Lc}(x)}{\longrightarrow} \operatorname{Lc}(1)\right)$, where the map $x: 1 \rightarrow X$ sends the element of the singleton 1 to the element $x$ of $X$. Thus, in order to verify that $\sum$ is locally monotone, it suffices to show that for every pair $f, g: \operatorname{Lc}(X) \rightarrow A$ with $f \leq g$ in Loc, we have $\bar{f} \leq \bar{g}$ in $\operatorname{Top}_{0}$. Let then $f$ and $g$ satisfy those conditions, that is, $f^{*} \geq g^{*}$ in Frm. Hence, for every $x \in X, \operatorname{Lc}(x) f^{*} \geq \operatorname{Lc}(x) g^{*}$ in Frm. Consequently, for every $a \in A$, the equality $\operatorname{Lc}(x) g^{*}(a)=1$ implies $\operatorname{Lc}(x) f^{*}(a)=1$. In other words, $\operatorname{Lc}(x) g^{*} \in \varphi(a)$ implies $\operatorname{Lc}(x) f^{*} \in \varphi(a)$. Since the topology of $\sum A$ is formed by all sets $\varphi(a)$, we have that, concerning the dual of the specialization order, $\operatorname{Lc}(x) f^{*} \leq \operatorname{Lc}(x) g^{*}$ for all $x \in X$. That is, $\bar{f} \leq \bar{g}$, as desired. In conclusion,

$$
\operatorname{Lc} \dashv \sum: \text { Loc } \rightarrow \text { Top }_{0}
$$

yields an adjunction with both functors locally monotone. Moreover, as it is well known, this adjunction induces an equivalence of categories between the full subcategory SpLoc of Loc of all spatial spaces and the full subcategory Sob of $\mathrm{Top}_{0}$ of all sober spaces.

Let us recall that a filter $\psi$ in $\Omega X$ is completely prime if, for every family $\left(U_{i}\right)_{i \in I}$ of open sets of $X$, the fact that $\bigcup_{i \in I} U_{i} \in \psi$ implies that $\psi$ contains some of the sets $U_{i}$. We recall that the functor $\Sigma:$ Loc $\rightarrow$ Top $_{0}$ may also be defined, up to isomorphism, by looking at $\sum L$ as the space whose points are the completely prime filters in $L$ and whose open sets are the sets $\Sigma_{a}, a \in L$, with $\Sigma_{a}$ consisting of all completely prime filters containing $a$.

Remark 5.3. We recall here the definition of the filter monad $\mathbf{T}=(T, \eta, \mu)$ over Top $_{0}$.

For every object $X, T X$ is the set of all filters in $\Omega X$ equipped with the topology generated by the sets

$$
\square U=\{\psi \in T X \mid U \in \psi\}, \quad U \in \Omega X .
$$

For every morphism $f: X \rightarrow Y$,

$$
T f(\psi)=\left\{V \in \Omega Y \mid f^{-1}(V) \in \psi\right\}, \psi \in T X .
$$

The unit $\eta$ and the multiplication $\mu$ are defined pointwisely by

$$
\eta_{X}(x)=\{U \in \Omega X \mid x \in U\} \quad \text { and } \quad \mu_{X}(\psi)=\{U \in \Omega X \mid \square U \in \psi\} .
$$

The filter monad $\mathbf{T}$ was studied by Day [6] and Wyler [33], who showed that the Eilenberg-Moore category of this monad is the category ContI of all continuous lattices and maps preserving all directed suprema and all infima (relative to the specialization order). Escardó $[7,8]$ observed that the filter monad is indeed a KZ-monad, and the $T$-embeddings are precisely the embeddings. 
Besides the filter monad T, Escardó and Flagg also analysed some submonads of the monad $\mathbf{T}([7,8,10])$. Namely they considered the proper filter monad and the prime filter monad. Here we are going to pay attention to the $n$-filter monad for every $n \geq 1$, according to the following definition.

Definition 5.4. Given a space $X$, a filter $\psi$ in $\Omega X$ is said to be $n$-prime provided that, for every family $\left(U_{i}\right)_{i \in k}$ of members of $\psi$ with $\operatorname{card}(k) \leq n$, if $\bigcup_{i \in k} U_{i}$ belongs to $\psi$ then $U_{j}$ belongs to $\psi$ for some $j$.

Remark 5.5. If in the above definition we put $n=1$ we obtain the definition of proper filter, that is, a filter not containing the empty set: just consider $\operatorname{card}(k)=0$. If we put $n=2$ then we obtain the definition of prime filter.

As it was shown in $[8,10]$, if, in Remark 5.3, we replace "filter" by "proper filter", or by "prime filter", we obtain also KZ-monads, the proper filter monad and the prime filter monad, respectively. Moreover, in the same manner we have the KZ-monad of the completely prime filters.

Notation 5.6. For $n \geq 1$ and $X \in \operatorname{Top}_{0}$, let $T_{n} X$ be the set of all $n$-prime filters on $\Omega X$ with the topology generated by the sets $\square U=\left\{\psi \in T_{n} X \mid U \in \psi\right\}, U \in \Omega X$. By defining the functor

$$
T_{n}: \mathrm{Top}_{0} \rightarrow \mathrm{Top}_{0}
$$

and the natural transformations $\eta_{n}$ and $\mu_{n}$ exactly as we defined, in Remark 5.3, $T, \eta$ and $\mu$, but considering always just $n$-prime filters instead of filters, we still obtain a KZmonad. This is figured out in an entirely analogous way to what was done in [10] for $n=1,2$, and is used in Theorem 5.9 below. Moreover, by imitating the techniques used in [10] for $n=1,2$, we conclude that the corresponding $T_{n}$-embeddings are precisely the $n$-flat embeddings, which we define next. First, let us recall that, as it is easy to see, a morphism $h: X \rightarrow Y$ is an embedding of $\operatorname{Top}_{0}$ iff the morphism $\operatorname{Lc}(h)=\left(h^{-1}\right)_{*}$, already described in Remark 3.12, is an embedding in Loc (see [17]).

Definition 5.7. In $\mathrm{Top}_{0}$, a morphism $h: X \rightarrow Y$ is said to be an $n$-flat embedding if the morphism $\operatorname{Lc}(h)$ is an $n$-flat embedding in Loc.

Notation 5.8. For every cardinal $n$, the image under $\sum$ of the subcategory $\mathcal{D}_{n}$ described in Notation 3.8 is a subcategory of $\operatorname{Top}_{0}$, let us denote it by $\mathcal{A}_{n}$,

$$
\mathcal{A}_{n}=\Sigma\left[\mathcal{D}_{n}\right]
$$

In particular, $\mathcal{A}_{1}$ is, up to isomorphism, the subcategory of Top $_{0}$ whose only non identity morphism is the inclusion $g_{0}:\{0\} \rightarrow S$, where $S$ is the Sierpiński space $\{0,1\}$ with $\{1\}$ the only nontrivial open set. We obtain $\mathcal{A}_{2}$, up to isomorphism, by adding to $\mathcal{A}_{0}$ the morphism $g_{2}: A_{2} \rightarrow S$, where $A_{2}=\{a, b, 0,1\}$ with the topology generated by $\{1\},\{a, 1\}$ and $\{b, 1\}$, and $g_{2}$ sends all elements of $A_{2}$ except 0 to 1 . 
In [5] we described dense embeddings and flat embeddings in terms of Kan-injectivity concerning a finite subcategory. In the following we obtain a similar characterization of $n$-embeddings, for every cardinal $n$, as a consequence of Theorem 3.10. Moreover, we use

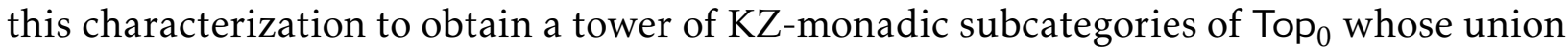
is the full subcategory of sober spaces.

Theorem 5.9. In $\mathrm{Top}_{0}$, for every cardinal $n \geq 1$, the class of $n$-flat embeddings is precisely $\mathcal{A}_{n}^{\text {KInj }}$, and the Kan-injective hull of $\mathcal{A}_{n}$ is the Eilenberg-Moore category of the KZ-monad $\mathbf{T}_{n}$. Moreover, the union of the chain of KZ-monadic subcategories $\mathbf{K}\left(\mathcal{A}_{n}\right)$ is the category Sob.

Proof. As we have shown in Remark 5.2, the two functors of the adjunction Lc $\dashv \sum$ : Loc $\rightarrow$ $\mathrm{Top}_{0}$ are locally monotone. Then, from Proposition 2.8 , we know that, for every cardinal $n, \Sigma f_{n}$ is (left) Kan-injective with respect to a morphism $h: X \rightarrow Y$ in Top $_{0}$ if and only if $f_{n}$ is Kan-injective with respect to $\operatorname{Lc}(h)$ in Loc. Consequently, it follows from Theorem 3.10 that $\mathcal{A}_{n}^{\mathrm{KInj}}$ is indeed the class of $n$-flat embeddings.

But $n$-flat embeddings coincide also with $T_{n}$-embeddings (see Notation 5.6 ). Then, by Proposition 2.7, the Eilenberg-Moore category of the $\mathrm{KZ}$-monad $\mathbf{T}_{n}$ is $\operatorname{KInj}\left(T_{n}\right.$-embeddings $)=$ $\operatorname{KInj}\left(\mathcal{A}_{n}^{\mathrm{KInj}}\right)=\mathbf{K}\left(\mathcal{A}_{n}\right)$.

It remains to show that the category Sob is the union of the categories $\mathbf{K}\left(\mathcal{A}_{n}\right)$. As observed in [10], Sob is the Eilenberg-Moore category of the KZ-monad $\overline{\mathbf{T}}=\Sigma \mathrm{Lc}$ of completely prime filters; and the $\bar{T}$-embeddings are the completely flat embeddings, i.e., those embeddings for which $\operatorname{Lc}(g)=\left(g^{-1}\right) *$ preserves arbitrary unions. Thus, from Proposition 2.7, it follows that Sob $=\operatorname{KInj}(\mathbb{C})$ for $\mathbb{C}$ the class of all completely flat embeddings. It is clear that every completely flat embedding is $n$-flat for every $n$. Then the inclusion of the categories $\mathbf{K}\left(\mathcal{A}_{n}\right)$ in Sob follows.

In order to show that Sob $\subseteq \mathbf{K}\left(\mathcal{A}_{n}\right)$ for some $n$, let $X$ be a sober space, and let $\Omega X$ have cardinality $n$. We show that $X \in \mathbf{K}\left(\mathcal{A}_{n}\right)$. Indeed, given any union $\cup_{i \in I} A_{i}$ of open sets of $X$, there is some $J \subseteq I$ such that $\operatorname{card}(J) \leq n$ and, for every $i \in I, A_{i}=A_{j}$ for some $j \in J$, in particular, $\cup_{j \in J} A_{j}=\cup_{i \in I} A_{i}$. Consequently any $n$-prime filter of $X$ is also completely prime. But then $\sum \operatorname{Lc}(X)=T_{n} X$. Since $X$ is sober, the map $\lambda_{X}: X \rightarrow \sum \operatorname{Lc}(X)$, where $\lambda$ is the unit of the adjunction $\operatorname{Lc}_{-} \perp$, is a homeomorphism. Being $\mathbf{K}\left(\mathcal{A}_{n}\right)$ closed under isomorphisms in $\mathrm{Top}_{0}$, we conclude that $X \in \mathbf{K}\left(\mathcal{A}_{n}\right)$. It remains to show that every continuous map between sober spaces belongs to $\mathbf{K}\left(\mathcal{A}_{n}\right)$ for some $n$. Let $f: Z \rightarrow W$ be a morphism in Sob. Let $n=\max \{\operatorname{card}(\Omega(Z)), \operatorname{card}(\Omega(W))\}$. Then both $Z$ and $W$ belong to $\mathbf{K}\left(\mathcal{A}_{n}\right)$, and $\lambda_{Z}$ and $\lambda_{W}$ are isomorphisms. Then $f=\eta_{Y}^{-1}\left(T_{n} f\right) \eta_{X}^{-1}$ and, since $\mathbf{K}\left(\mathcal{A}_{n}\right)$ is closed under isomorphisms, it contains $f$.

Corollary 5.10. Sob is the Kan-injective hull of the union of all categories $\mathcal{A}_{n}$ both in Top 0 and in Sob.

Proof. The union of all subcategories $\mathcal{A}_{n}$ is also a category, and it is contained in Sob (since the image of $\Sigma$ is contained in Sob). Let us denote it by $\mathcal{A}$. Then, in Top 0 , denoting the 
class of all completely flat embeddings by $\mathbb{C}$, we have

$$
\mathcal{A}^{\mathrm{KInj}}=\left(\bigcup_{n \in \text { Card }} \mathcal{A}_{n}\right)^{\mathrm{KInj}}=\bigcap_{n \in \text { Card }} \mathcal{A}_{n}^{\mathrm{KInj}}=\mathbb{C} .
$$

But we know that Sob is the Eilenberg-Moore category of the completely prime filter monad $\overline{\mathbf{T}}$, being the completely flat embeddings the corresponding $\overline{\mathbf{T}}$-embeddings. Consequently, by Proposition 2.7, $\operatorname{Sob}=\mathbf{K}(\mathcal{A})$.

Also the Kan-injective hull of $\mathcal{A}$ in Sob is Sob as we show now. We use the index Sob to specify that we are working in the category Sob; otherwise we are referring to $\mathrm{Top}_{0}$. Indeed, since Sob is full in $\operatorname{Top}_{0}$ we have that $\operatorname{KInj}_{\text {Sob }}\left(\mathcal{A}^{\left.\mathrm{KInj} j_{\text {Sob }}\right)}=\operatorname{KInj}\left(\mathcal{A}^{\mathrm{KInj} \text { Sob }}\right) \cap \operatorname{Sob}\right.$. Then, since $\mathrm{K}(\mathcal{A})=\operatorname{KInj}\left(\mathcal{A}^{\mathrm{KInj}}\right)=\mathrm{Sob}$, and $\mathcal{A}^{\mathrm{KInj}} \mathrm{Sob} \subseteq \mathcal{A}^{\mathrm{KInj}}$, we obtain the desired equality:

$$
\mathrm{Sob}=\mathrm{K}(\mathcal{A}) \cap \mathrm{Sob} \subseteq \operatorname{KInj}\left(\mathcal{A}^{\mathrm{KInj} \text { Sob }}\right) \cap \mathrm{Sob}=\operatorname{KInj}_{\mathrm{Sob}}\left(\mathcal{A}^{\mathrm{KInj} \text { Sob }}\right)=\mathrm{K}_{\mathrm{Sob}}(\mathcal{A}) .
$$

Remark 5.11. The fact that $\mathbf{K}\left(\mathcal{A}_{1}\right)$ and $\mathbf{K}\left(\mathcal{A}_{2}\right)$ are precisely the categories of EilenbergMoore algebras of the proper filter monad and the prime filter monad, respectively, was proved in [5]. However, there the morphism $f_{0}$ of $\mathcal{A}_{1}$, used to characterize the dense embeddings, was different; moreover, this morphism was wrongly missing in the description of the category $\mathcal{A}_{2}$.

Remark 5.12. Let ScottDI be the category of Scott domains and functions which preserve directed suprema and non-empty infima, which is a subcategory of $\mathrm{Top}_{0}$ via the Scott topology ([12]). This category was proved to be the Eilenberg-Moore category of the proper filter monad by Wyler [33]. Thus $\mathbf{K}\left(\mathcal{A}_{1}\right)=\mathbf{S c o t t D I}$.

The Eilenberg-Moore category of the prime filter monad was characterized by Simmons [28] and Wyler [32], it is the subcategory SComp of Top S $_{0}$ all stably compact spaces and stably continuous maps. That is, the objects of SComp are those spaces which are sober, locally compact and whose family of all saturated compacts is closed under finite intersections, and the morphisms are the continuous maps such that for every pair of open sets $U$ and $V$ and a compact set $K$ with $U \subseteq K \subseteq V$ in $Y$, there exists some compact $K^{\prime}$ in $X$ fulfilling $f^{-1}(U) \subseteq K^{\prime} \subseteq f^{-1}(V)$. From the above theorem, $\mathbf{K}\left(\mathcal{A}_{2}\right)=$ SComp. In [14] Hofmann gave a characterization of the Eilenberg-Moore category for every monad $\mathbf{T}_{n}$, which generalizes the one of SComp described in Remark 5.12.

We also recall from [5] that the Kan-injective hull of the Sierpiński space is the category ContI of continuous lattices and maps that preserve directed suprema and arbitrary infima.

\section{REFERENCES}

[1] J. Adámek, M. Hébert and L. Sousa, The orthogonal subcategory problem and the small object argument, Appl. Categ. Structures 17 (2009), 211-246. 
[2] J. Adámek, H. Herrlich and G. E. Strecker, Abstract and concrete categories, John Wiley and Sons, New York 1990, Repr. Theory Appl. Categ. 17 (2006), 1-507.

[3] J. Adámek, L. Sousa, J. Velebil, Kan injectivity in order-enriched categories, Math. Structures Comput. Sci. 25 (2015), no. 1, 6-45.

[4] B. Banaschewski, Projective frames: a general view, Cah. Topol. Géom. Différ. Catég. 46 (2005), no. $4,301-312$.

[5] M. Carvalho and L. Sousa, Order-preserving reflectors and injectivity, Topology Appl. 158 (2011), no. $17,2408-2422$.

[6] A. Day, Filter monads, continuous lattices and closure systems, Canad. J. Math. 27 (1975), 50-59.

[7] M. H. Escardó, Injective spaces via the filter monad, Proceedings of the 12th Summer Conference on General Topology and its Applications, 1997.

[8] M. H. Escardó, Properly injective spaces and function spaces, Topology Appl. 89(1-2) (1998), 75120.

[9] M. H. Escardó, Injective locales over perfect embeddings and algebras of the upper powerlocale monad, Appl. Gen. Topol. 4(1) (2003), 193-200.

[10] M. H. Escardó and R. C. Flagg, Semantic domains, injective spaces and monads, Electron. Notes Theor. Comput. Sci. 20 (1999).

[11] P. J. Freyd and G. M. Kelly, Categories of continuous functors I, J. Pure Appl. Algebra 2 (1972), $169-191$.

[12] G. Gierz, K. H. Hofmann, K. Keimel, J. D. Lawson, M. W. Mislove and D. S. Scott, A Compendium of Continuous Lattices, Springer-Verlag, Berlin-New York, 1980.

[13] R.-E. Hoffmann, Charakterisierung nüchterner Räume, Manuscripta Math. 15 (1975), 185-191.

[14] D. Hofmann, A four for the price of one duality principle for distributive topological spaces, Order 30 (2013), n 2, 643-655.

[15] S. S. Hong, Extensive subcategories of the category of $T_{0}$-spaces, Canad. J. Math. 27, 311-318.

[16] P. T. Johnstone, The Gleason cover of a topos II, J. Pure Appl. Algebra 22 (1981), 229-247.

[17] P. T. Johnstone, Stone Spaces, Cambridge University Press, Cambridge, 1982.

[18] P. T. Johnstone, Sketches of an Elephant: A Topos Theory Compendium, vol. 2. Number 44 in Oxford Logic Guides, The Clarendon Press, Oxford University Press, Oxford, 2002.

[19] G. M. Kelly, A unified treatment of transfinite constructions for free algebras, free monoids, colimits, associated sheaves, and so on, Bull. Austral. Math. Soc. 22 (1980), 1-83.

[20] G. M. Kelly, Basic concepts of enriched category theory, London Math. Soc. Lecture Notes Series 64, Cambridge Univ. Press, 1982, Repr. Theory Appl. Categ. 10 (2005), 1-136.

[21] A. Kock, Monads for which structures are adjoint to units (version 3), J. Pure Appl. Algebra 104 (1995), 41-59.

[22] S. Mac Lane, Categories for the working mathematician, 2nd edition, Springer, 1998.

[23] S. Mantovani, Epireflective hulls of spaces of ordinals in $T_{0}$, Quaestiones Math. 7 (1984), 203211.

[24] L. D. Nel and R. G. Wilson, Epireflections in the category of $T_{0}$-spaces, Fund. Math. LXXV (1972), 69-74.

[25] J. Picado, A. Pultr, Frames and Locales: Topology Without Points, Birkhäuser/Springer Basel AG, Basel, 2012.

[26] G. Raney, A subdirected union representation of completely distributive complete lattices, Proc. Amer. Math. Soc. 4 (1953), 518-522.

[27] D. S. Scott, Continuous lattices, in: Toposes, algebraic geometry and logic (F. W. Lawvere, ed.), Lecture Notes in Mathematics, vol. 274, Springer Verlag, 1972, 97-136. 
[28] H. Simmons, A couple of triples, Topology Appl. 13 (1982) 201-223.

[29] L. Sousa, $\alpha$-sober spaces via the orthogonal closure operator, Appl. Categ. Structures 4 (1996), $87-95$.

[30] M.B. Smyth, Stable compactification I, J. London Math. Soc. (2) 45 (1992), no. 2, 321-340.

[31] M. B. Smyth and G. D. Plotkin, The category-theoretic solution of recursive domain equations, SIAM J. Comput. 11 (1982), 761-783.

[32] O. Wyler, Compact ordered spaces and prime Wallman compactifications. In: Categorical Topology, 618-635, Sigma Ser. Pure Math., 5, Heldermann, Berlin, 1984.

[33] O. Wyler, Algebraic theories for continuous semilattices, Arch. Rational Mech. Anal. 90 (1985), no. 2, 99-113.

[34] V. Zöberlein, Doctrines on 2-categories, Math. Z. 148 (1976), 267-279.

isCAC, Polytechnic Institute of Coimbra, Portugal

E-mail address: margaridacarva@gmail.com

Centre for Mathematics of the University of Coimbra \& Polytechnic Institute of Viseu, Portugal E-mail address, corresponding author: sousa@estv.ipv.pt 